\title{
Enabling Reflective \& Refractive Depth Representation in Computer-Generated Holography
}

\author{
Aaron Demolder \\ Hammadi Nait-Charif \\ Valery Adzhiev \\ Bournemouth University \\ Bournemouth, UK
}

\author{
Alfred Newman \\ Tom Durrant \\ Andrzej Kaczorowski \\ VividQ Ltd \\ Cambridge, UK
}

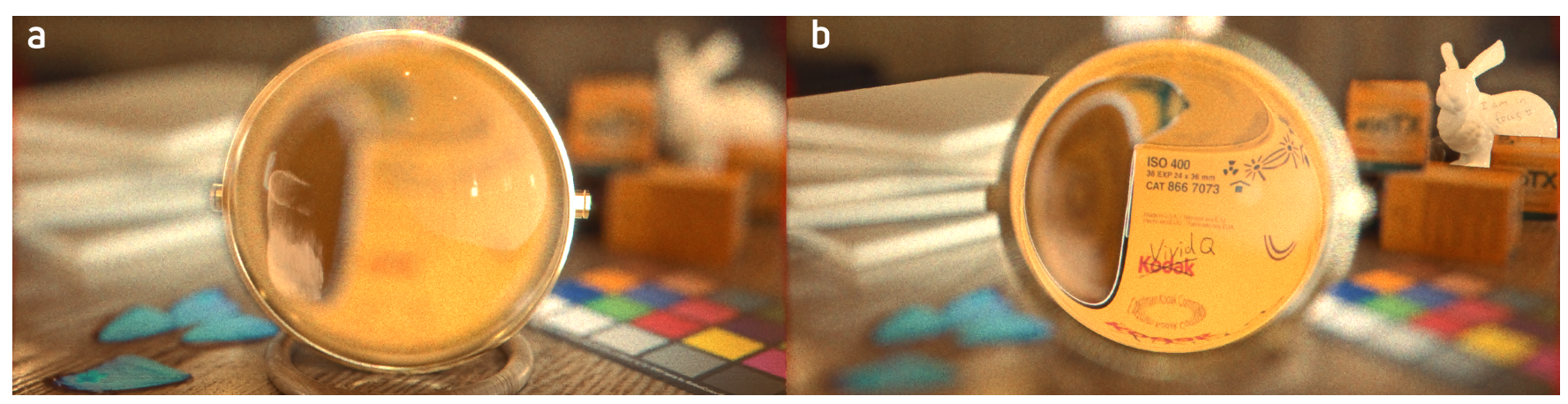

Figure 1: Holographic replays (simulated) of a scene containing a lens of $f=180 \mathrm{~mm}, 0.5 \mathrm{~m}$ from camera. (a) Focus is at $0.46 \mathrm{~m}$ on the smudge, reflection focus of lights is $0.52 \mathrm{~m}$. (b) Focus (1m) now on the text and bunny, the film box is $150 \mathrm{~mm}$ behind the lens.

ACM Reference Format:
Aaron Demolder, Hammadi Nait-Charif, Valery Adzhiev, Alfred Newman, Tom Durrant, and Andrzej Kaczorowski. 2021. Enabling Reflective \& Refractive Depth Representation in Computer-Generated Holography . In Special Interest Group on Computer Graphics and Interactive Techniques Conference Posters (SIGGRAPH '21 Posters), August 09-13, 2021. ACM, New York, NY, USA, 2 pages. https://doi.org/10.1145/3450618.3469177

\section{INTRODUCTION}

Computer-Generated Holography (CGH) is a technique for the reconstruction and display of three-dimensional imagery through diffraction and interference of light. Holograms are generated digitally by calculating light propagation, and displayed on devices called Spatial Light Modulators (SLMs). When illuminated by coherent laser light, holograms then replay a full 3D scene. Recently, holography has received significant attention from industrial and academic communities, with neural holography [Peng et al. 2020] [Shi et al. 2021] and numerous other techniques [Sahin et al. 2020]. Applications of holography include Augmented/Virtual Reality [Widjanarko et al. 2020], Head-Up Displays, and larger display devices [An et al. 2020]. In the real world, objects can be viewed in reflections (such as mirrors) and through refractions (such as glass or water); the depth at which these objects focus is defined by the focal power of the material. In computer graphics, realistic

Permission to make digital or hard copies of part or all of this work for personal or classroom use is granted without fee provided that copies are not made or distributed for profit or commercial advantage and that copies bear this notice and the full citation on the first page. Copyrights for third-party components of this work must be honored. For all other uses, contact the owner/author(s).

SIGGRAPH '21 Posters, August 09-13, 2021, Virtual Event, USA

(C) 2021 Copyright held by the owner/author(s).

ACM ISBN 978-1-4503-8371-4/21/08.

https://doi.org/10.1145/3450618.3469177 representation of materials is essential to the realism of the image. These effects do not pose an issue for $2 \mathrm{D}$ display, as only the pixel intensities are required while focus is pre-determined. By contrast, in image/layer-based holography, z-depth values are used that correspond to the focus distance of objects. While this technique works well for the first object hit by the raytracer, it is unable to accurately encode the depth at which virtual objects appear after reflection or refraction. It is hence necessary for the renderer to pass multiple depth values to the hologram generation engine to ensure realistic representation of reflections and refractions in a realistic $3 \mathrm{D}$ display.

\section{APPROACH}

We propose a method of utilising additional depth information required for reflective and refractive material representations on holographic displays via multiple render passes, and a first-of-its-kind method of multi-pass holographic compositing whereby render passes are combined in the hologram (frequency) domain. The resulting hologram successfully represents the input render, but now with the correct depth information for all render passes supplied. The resulting hologram can consequently be displayed on an SLM and viewed by eye with the correct perceptual cues, resulting in correct focus properties (see Figure 1).

\section{IMPLEMENTATION}

The 'depth' of a point refers to the distance from which multiple rays from that point appear to diverge. In the case of an object being viewed in the reflection of a flat mirror, the total depth is the length from the object to the mirror and then to the viewer. Figure 2(a) shows the primary ray from viewer to mirror, and a secondary ray [Whitted 1980] from mirror to object. A z-depth pass will provide the length of only the primary ray, so must be added to the length 


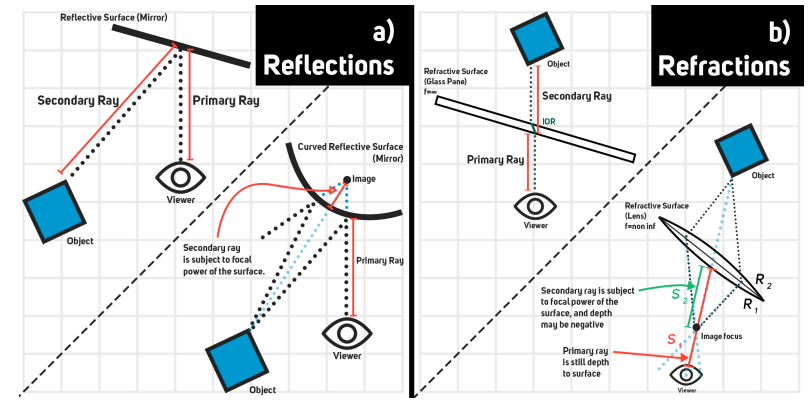

Figure 2: (a) The total depth required for reflections is the summation of primary and secondary rays, following the law of reflection. (b) The focal length is calculated with the Index of Refraction (IOR) given by Snell's Law, and the thickness of the material. A material may exhibit both reflective and refractive properties simultaneously.

of the reflection secondary ray for the correct depth of the object in camera space. A refraction depth pass is performed in a similar fashion, as seen in Figure 2(b). In the case of looking through glass, the primary ray is formed by the depth from viewer to the surface of the glass, but the length of the secondary ray is determined by the shape of the surface as well as the refractive index. The resulting image may be real or virtual, and have magnification. The focal length of the lens is given by the lensmaker's equation:

$$
\frac{1}{f}=(n-1)\left(\frac{1}{R_{1}}-\frac{1}{R_{2}}\right)
$$

Where $f$ is focal length, $\mathrm{n}$ is Index of Refraction (IOR) and $R_{1}, R_{2}$ are the radii of curvature of the surface. The new secondary ray depth of the object image visible through the lens is given by:

$$
\frac{1}{S_{2}}=\frac{1}{f}-\frac{1}{S_{1}}
$$

Where $S_{1}$ and $S_{2}$ are the depths to the lens and the object respectively. When added, $S_{1}$ and $S_{2}$ provide the total correct depth value. Most renderers will already store a value for focus depth per pixel per render pass. Each colour pass can still be adjusted as required in compositing software. The resulting passes can then be loaded for hologram generation - here we employ VividQ's Software Development Kit. The resulting outputs are images in Fourier space (where the image is decomposed into its constituent frequencies) for each render pass which can be displayed on a holographic projector. As holograms are additive in the same way light is - each of the holograms can consequently be composited using a familiar multi-pass process. Whilst still in frequency space, each of the holograms are combined, shown in Figure 3. When displayed on an SLM, this will 'replay' the 3D scene to the viewer, with the correct material depth information.

\section{CONCLUSIONS}

The results shown in both Figure 1 and Figure 4 demonstrate that the methods introduced represent a significant step towards realistic imagery for holographic display, with physically correct reflections and refractions - whilst remaining fully compatible with the current computer graphics pipeline. However, Image-based hologram generation methods result in black artefacts in a scene, as no colour data exists behind each depth layer - this is visible when objects defocus. This could be minimised by including data that

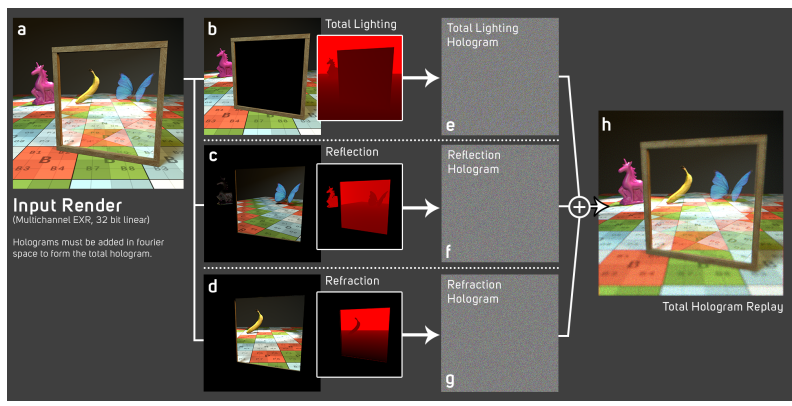

Figure 3: (a) The input multichannel file. (b) Total Lighting render, z-depth. (c) Reflection render, associated depth. (d) Refraction render, associated depth. (e) Hologram of Total Lighting only. (f) Hologram of Reflection only. (g) Hologram of Refraction Only. (h) The total composite hologram replay, via a plus operation of the holograms for each render pass.

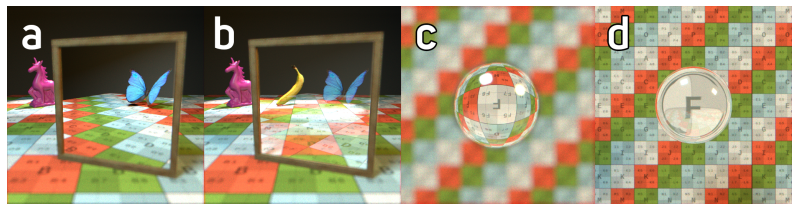

Figure 4: (a) Replay of butterfly reflection and unicorn (both $9 \mathrm{~m}$ ) (b) Changing the mirror to glass reveals a banana $(9 \mathrm{~m})$. (c) Grid at $1 \mathrm{~m}$ from camera focused by a lens $(f=100 \mathrm{~mm})$ placed $850 \mathrm{~mm}$ from the camera, the grid focuses at $550 \mathrm{~mm}$ (real). Reflection focus is $950 \mathrm{~mm}$. (d) Grid at $1 \mathrm{~m}$ from camera focused by a lens $(f=100 \mathrm{~mm})$ placed $950 \mathrm{~mm}$ from the camera, the grid focuses at $1050 \mathrm{~mm}$ (virtual). Reflection focus is $1050 \mathrm{~mm}$.

allows object-based and render-pass-based overscan for objects that are occluded. For best results this data would be sourced directly from the renderer, but estimates could be made with object based AOVs (such as cryptomatte [Friedman and Jones 2015] or object ID manifests [Hillman 2018]) by generating dilated edges per object.

\section{ACKNOWLEDGMENTS}

This work has been supported by grant funding of the Centre for Digital Entertainment by the Engineering and Physical Sciences Research Council (EPSRC) EP/L016540/1 and VividQ Ltd.

\section{REFERENCES}

Jungkwuen An, Kanghee Won, Young Kim, Jong-Young Hong, Hojung Kim, Yongkyu Kim, Hoon Song, Chilsung Choi, Yunhee Kim, Juwon Seo, Alexander Morozov, Hyunsik Park, Sunghoon Hong, Sungwoo Hwang, Kichul Kim, and Hong-Seok Lee. 2020. Slim-panel holographic video display. Nature Communications 11, 1 (2020). https://doi.org/10.1038/s41467-020-19298-4

Jonah Friedman and Andrew C. Jones. 2015. Fully Automatic ID Mattes with Support for Motion Blur and Transparency. In ACM SIGGRAPH 2015 Posters (SIGGRAPH '15). Article 47. https://doi.org/10.1145/2787626.2787629

Peter Hillman. 2018. A Scheme for Storing Object ID Manifests in OpenEXR Images. In Proceedings of the 8th Annual Digital Production Symposium (DigiPro '18). Article 9. https://doi.org/10.1145/3233085.3233086

Yifan Peng, Suyeon Choi, Nitish Padmanaban, and Gordon Wetzstein. 2020. Neural Holography with Camera-in-the-Loop Training. ACM Trans. Graph. 39, 6, Article 185 (2020). https://doi.org/10.1145/3414685.3417802

Erdem Sahin, Elena Stoykova, Jani Mäkinen, and Atanas Gotchev. 2020. ComputerGenerated Holograms for 3D Imaging: A Survey. ACM Comput. Surv. 53, 2, Article 32 (2020). https://doi.org/10.1145/3378444

Liang Shi, Beichen Li, Changil Kim, Petr Kellnhofer, and Wojciech Matusik. 2021. Towards real-time photorealistic 3D holography with deep neural networks. Nature 591, 7849 (2021). https://doi.org/10.1038/s41586-020-03152-0

Turner Whitted. 1980. An Improved Illumination Model for Shaded Display. Commun. ACM 23, 6 (1980). https://doi.org/10.1145/358876.358882

T. Widjanarko, M. El Guendy, A. O. Spiess, D. M. Sullivan, T. J. Durrant, O. A. Tastemur, A. J. Newman, D. F. Milne, and A. Kaczorowski. 2020. Clearing key barriers to mass adoption of augmented reality with computer-generated holography. In Optical Architectures for Displays and Sensing in Augmented, Virtual, and Mixed Reality (AR, VR, MR), Vol. 11310. SPIE. https://doi.org/10.1117/12.2544979 